\title{
A microprocessor system for the digital synthesis of pulsed and continuous discharges of electric fish (or animal vocalizations)
}

\author{
Bernd Kramer ${ }^{1}$ and Dieter Weymann ${ }^{2}$ \\ ${ }^{1}$ Zoological Institute and ${ }^{2}$ Department of Biology of the University, Regensburg (F.R.G.) \\ (Received 11 June 1986) \\ (Revised version received 13 October 1986) \\ (Accepted 14 October 1986)
}

\begin{abstract}
Key words: Animal communication signal; Animal orientation signal; Electric organ discharge; Microprocessor; Digital synthesis; Analogue-to-digital conversion; Digital-to-analogue conversion; Signal waveform
\end{abstract}

\begin{abstract}
Traditionally, the sensitivity of electric fish to electric stimuli has been investigated using a variety of electronically generated signal functions, for example sine, square, and sawtooth waves (both continuous and pulsed). None of these resemble the speciesor sex-specific electric organ discharges (EODs) of fish. Therefore, EODs recorded on magnetic tape were used in open-loop playback experiments. However, as shown in the present paper, the waveforms of EODs reproduced from magnetic tape are distorted, and cannot be manipulated with speed and accuracy as to frequency or pulse repetition rate by feedback from the experiment. Good EOD imitations are generated by our microprocessor-based system for the digital synthesis of EODs which is controlled by a small 'personal computer'. Digitized EOD samples edited by computer are output by the fast digital-to-analogue converter of the microprocessor system. This system was devised for the synthesis of pulse-type (discontinuous) and wave type (continuous) EODs with continuous, on-line frequency and amplitude control. The rate of digital synthesis is $500 \mathrm{kHz}$ in pulse-type EODs, and $250-500 \mathrm{kHz}$ in wave type EODs at $0.06-0.24 \mathrm{~Hz}$ frequency resolution, depending on the repetition frequency of the synthesized wave EOD. The present paper describes the steps in EOD synthesis, and indicates some applications which benefit from the playback of high-fidelity imitations of natural EODs at amplitudes and frequencies selected by the experimenter, or automatically controlled by feedback from the experiment in closed-loop applications.
\end{abstract}

\section{INTRODUCTION}

In the study of the electric sense of lower vertebrates (for a review see ref. 2) only simple stimuli were used until recently, that is waveforms available from function or pulse generators (such as sine, square, sawtooth, and triangle waves, or monopolar and bipolar square wave pulses). None of these resemble the electric organ discharges (EODs) generated by electric fish (for example, the South American Gymnotiformes or knife fishes, or the African Mormyriformes). EOD waveforms of sympatric groups of electric fish are species-specific with little overlap among species (see, for example, ref. 17), or, in certain species, sexually dimorphic ${ }^{13,15}$. Since Scheich's ${ }^{20}$ electroreceptor study which demonstrated phasesensitive, tuned T-electroreceptors (in addition to phase-insensitive, tuned P-receptors) in the knifefish Eigenmannia, the hypothesis of mate selection by time pattern cues of EOD waveforms ${ }^{7,9}$ was supported by a putative sensory mechanism. In order to find out whether, in fact, temporal (time domain), or spectral (frequency domain) prop-

Correspondence: B. Kramer, Zoological Institute of the University, D-8400 Regensburg, F.R.G. 
erties of EODs provide the cues for mate recognition ${ }^{15}$, a method was needed to generate any waveform, especially those of natural EODs, with high fidelity.

Attempts to make electronically generated signal functions look like natural EOD waveforms included their deliberate distortion by electronic filtering. Examples are: Westby's study ${ }^{24}$ of Gymnotus carapo with its pulse-type EOD (that is, EODs separated by relatively long intervals of silence), and Gottschalk's study ${ }^{4}$ of Eigenmannia with its wave-type EOD (that is, quasi-sinusoidal EODs of extremely stable frequency); both authors used square wave pulses distorted by RC-circuits or similar. However, these and other attempts did not give satisfactory results.

The play-back of EODs recorded on magnetic tape $^{9}$ was also unsatisfactory for two reasons. (1) Because of limitations of present magnetic tapes, EOD waveforms are distorted (see Figs. 1b, 2; see also ref. 18). This problem is especially severe in the very short, click-like EOD pulses of broad spectral composition found in many African Mormyridae and South American Gymnotidae, but is also present in wave EODs (especially those of low fundamental frequency and high harmonic content as observed, for example, in Eigenmannia males (see Fig. 6 and ref. 15). (2) When playing back tape-recorded EODs the repetition rate of pulse EODs, or the frequency of wave EODs, cannot be manipulated with speed and accuracy in response to feedback from the EOD of a stimulated fish, precluding the use of magnetic tape-recorders in closed-loop experiments. Play-back of tape-recorded EODs is still a useful technique in open-loop applications focussing on the inter-EOD time interval patterns (of, for example, mormyrids, which are speciesspecific and communicate motivational state $^{12,16}$ ), using electronically generated pulse waveforms (such as single sine wave pulses) triggered from the recorded EODs.

A more promising approach was the use of an analogue-to-digital (A/D) converted waveform for output by the digital-to-analogue $(D / A)$ converter of a minicomputer (for example, the fairly slow 2-ms pulse of Hypopomus artedi ${ }^{7}$ ). The drawback is that even powerful minicomputers
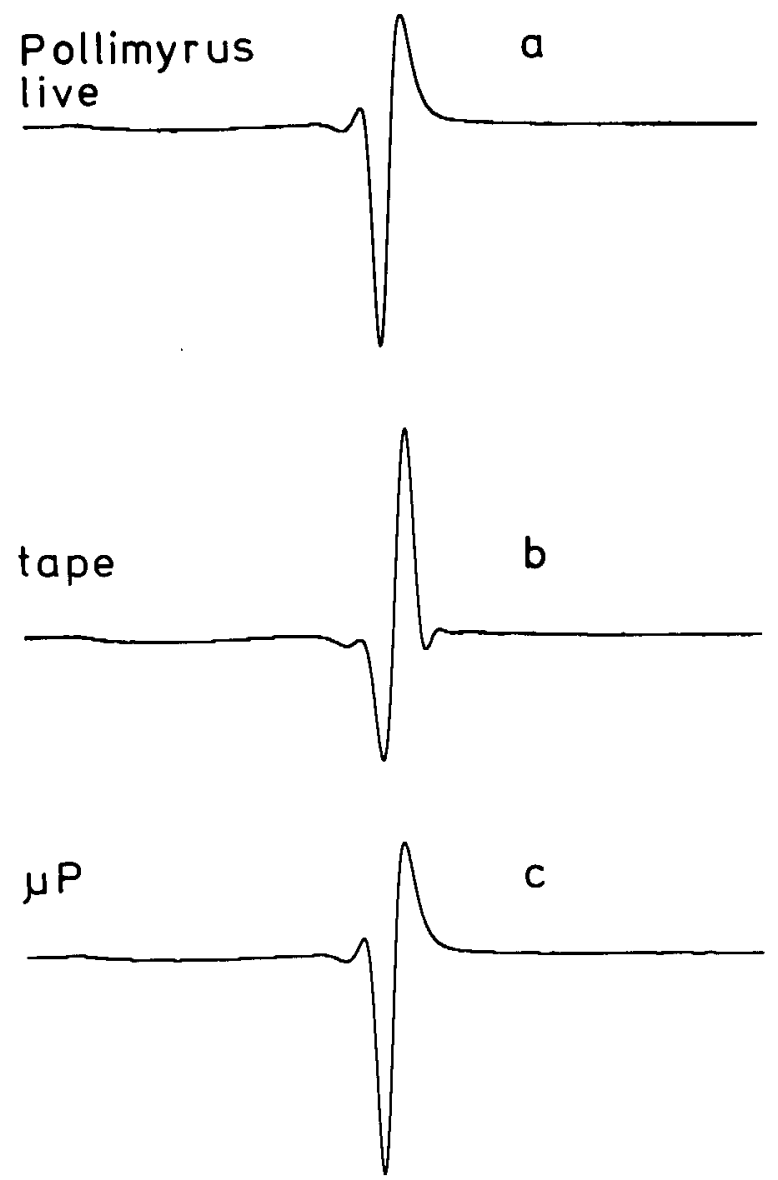

Fig. 1. Comparison of the digitized pulse EOD of a Pollimyrus isidori (a, live) with a playback from magnetic tape (b, tape), and with a digitally synthesized $\operatorname{EOD~(c,~} \mu \mathrm{P})$. In all traces head-positive is up (linear amplitudes in volts); each trace represents $0.8 \mathrm{~ms}$. Note that the waveform of the taped pulse is severely distorted and rings. The taped pulse (b) shows 6 phases instead of Pollimyrus' 4 phases (a, the first phase is the weak head-negative potential); the main phase of the taped pulse (b) is a large head-positive potential instead of the head-negative main spike seen in ' $a$ '. EOD waveform is fully retained in 'c'. All pulses were digitized with an 8-bit, 2-MHz transient recorder. The recordings ( $a, b)$ are from the same individual within a few minutes and under identical conditions. The EOD sample ' $a$ ' served for synthesis of the EOD imitation ' $c$ '.

(which are - despite their name - large and expensive systems) are too slow in sampling and outputting voltages (typically $20 \mathrm{kHz}$ sampling and conversion rate, with up to $50 \mathrm{kHz}$ only in the most powerful systems, such as Digital Equipment Corporation's PDP 11/40). For example, Pollimyrus isidori's $50 \mu \mathrm{s}$ EOD (without slower 


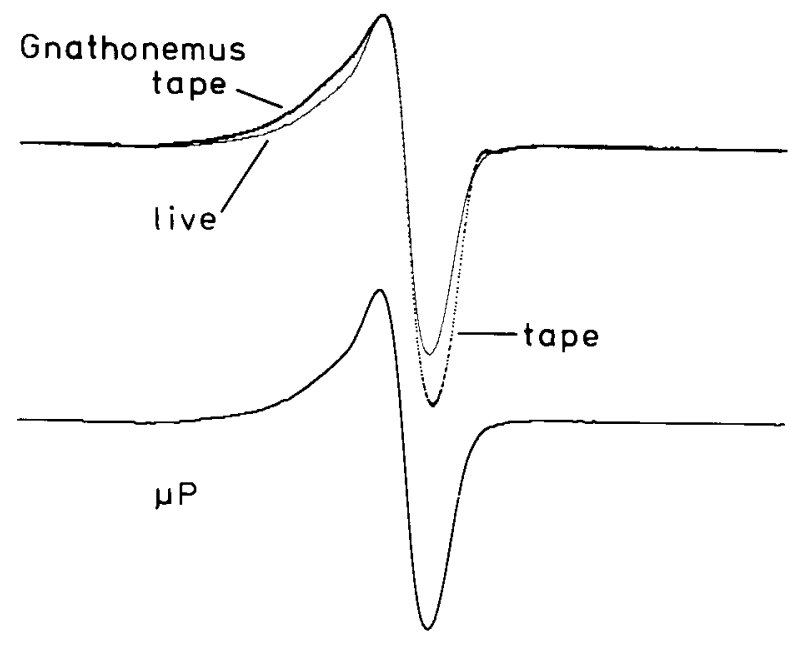

Fig. 2. Comparison of the digitized pulse EOD of Gnathonemus petersii with a playback from magnetic tape, and with a digitally synthesized EOD. Recording conditions and scale and trace conventions as in Fig. 1. The EODs are normalized to the same height of the head-positive peak, and to the same $\mathrm{X}$-axis position of the zero-crossing of the fast head-negative transient. Top: Although much slower than the Pollimyrus EOD, Gnathonemus' EOD is still considerably distorted by recording on tape, and the head-negative peak is slightly too late. The dotted waveform represents the individual $\mathrm{A} / \mathrm{D}$ data points of the digitized EOD stored on magnetic tape; the dots merge at slow rates of voltage change. The digitized EOD of the live fish is shown in the solid trace. Bottom: $\mu \mathrm{P}$-generated waveform synthesized from the EOD shown in the solid trace (top).

pre- and post-potentials of low amplitude; Fig. 1a) would be represented by only 2 voltage levels even at $50 \mathrm{kHz}$ sampling rate. Therefore, this method yielded synthetic EODs unacceptably distorted in all but the longest pulse EODs.

Wave EODs have not yet been synthesized, since in addition to the technical speed problem, there is the difficulty of continuous voltage change with no intervals of silence between EODs. In pulse EODs, the digitally stored data can be directly used for triggered output because of the long intervals of silence between pulses; in wave EODs this is not possible.

For these reasons we designed a microprocessor-based system controlled by a small 'personal computer' for the high-speed synthesis of pulse and wave EODs, with on-line control of the microprocessor for closed-loop experiments.
The following description includes the necessary detail for the construction of the system; since all parts or techniques are standard they may be replaced by equivalent or improved components and techniques which are locally available. The computer programs in BASIC are short and simple (and machine-specific); therefore, an overview of the procedures employed is presented, rather than a host of technical detail.

\section{MATERIALS AND METHODS}

\section{Hardware}

The central component of our system is a small Hewlett-Packard model 85A 'personal computer' (PC) with 32 kbytes of memory, and a dual floppy disc drive (540 kbytes of storage). A Vuko model VK $12-2$ transient recorder (8 bit, $2 \mathrm{MHz} ; 2000$ memory addresses, or words) records the EODs (see below) as a series of 8-bit integer numbers which are stored on disc for later editing. D/A-converted waveforms are viewed on an oscilloscope; hard-copy output (via an IEEEinterface) is produced on a Hewlett-Packard model $9872 \mathrm{~A}$ digital plotter controlled by the computer.

Our microprocessor-based system ( $\mu \mathrm{P}$; Fig. 3 ) for the synthesis of EODs was built by the second author (D.W.). It consists of an RCA (Radio

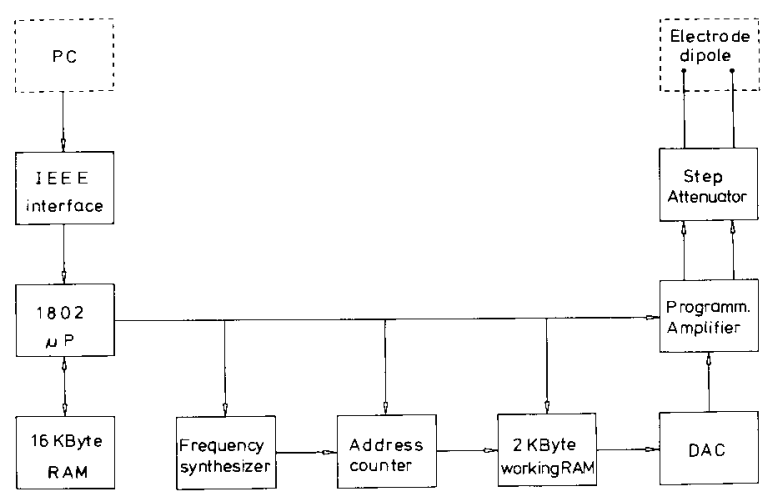

Fig. 3. Block diagram of the components of a microprocessor $(\mu \mathrm{P})$-based system for the digital synthesis of EODs (the PC, personal computer, is not part of the system, but controls it via an IEEE-interface which is common to the $\mathrm{PC}$ and the $\mu \mathrm{P}$ system). The electrode dipole is the output device (which can be replaced by other transducers in other applications). 
Corporation of America) type CDP 1802 microprocessor with 16 kbyte Random Access Memory (RAM; expandable to 64 kbyte RAM) backed up by rechargeable batteries. The first kbyte stores a machine-code operating system, and data specifying a particular job, such as frequency and amplitude. The remaining memory stores up to 7 EOD waveforms (as lists of 2048 8-bit-integers), or up to 6782 time intervals (as 16-bit-integers), or any combination thereof which does not exceed total memory. For generating analogue waveforms the interesting EOD data are copied into a separate working RAM $(2048 \times 8$-bit $)$. An address counter sequentially outputs the contents of the working RAM to a D/A-converter. This is quite similar to the operating principle of a transient recorder. Gates at the input of the address counter select address zero for start and stop of the cyclical outputting process.

The address counter is advanced by clock pulses generated by a quartz-stabilized, programmable frequency synthesizer (12-bit frequency resolution). Maximum frequency is 500 $\mathrm{kHz}$, minimum frequency which equals the frequency resolution is $122 \mathrm{~Hz}$. This yields a repetition frequency of up to $244 \mathrm{~Hz}$ (with 0.06 $\mathrm{Hz}$ resolution) for the cyclical output of the whole working RAM, that is, the analogue signal such as an EOD is synthesized at a frequency of up to $244 \mathrm{~Hz}$. Higher frequencies of the analogue signal are achieved by storing two or more identical signal cycles in the working RAM. More details about the D/A-converter, the programmable output amplifiers, and the attenuators are given in section IV, EOD output.

The $\mu \mathrm{P}$ is connected to the PC by an IEEEinterface which occupies two memory locations (internally selectable). One address serves for loading the operating system from the PC (stored as a disc file), the other serves for accepting data and instructions. Maximum block transfer rate is $13.4 \mathrm{kbytes} / \mathrm{s}$.

Our system is highly flexible by virtue of its modular design and software programming. By adding or changing modules, or by modifying the operating system, the system can be adapted for the most diverse applications.

EODs were recorded with a differential amplifier $(1 \mathrm{~Hz}-100 \mathrm{kHz})$ with the positive electrode near the head, the negative electrode near the tail of the fish (Fig. 4). EODs were stored using either a transient recorder (see above, Fig. 4), or a magnetic tape recorder. A high-quality Nagra IV-SJ tape recorder was used for EOD recordings on magnetic tape (Figs. $1 \mathrm{~b}$ and 2, dotted trace). Tape speed was a maximum $38.1 \mathrm{~cm} / \mathrm{s}$ during recording and playback $( \pm 1.0 \mathrm{~dB}$ from $25 \mathrm{~Hz}$ to $35 \mathrm{kHz}$; direct recording because of the high frequency content of pulse EODs). Water conductivity was $100 \mu \mathrm{S} / \mathrm{cm}$, temperature was $27.0^{\circ} \mathrm{C}$.

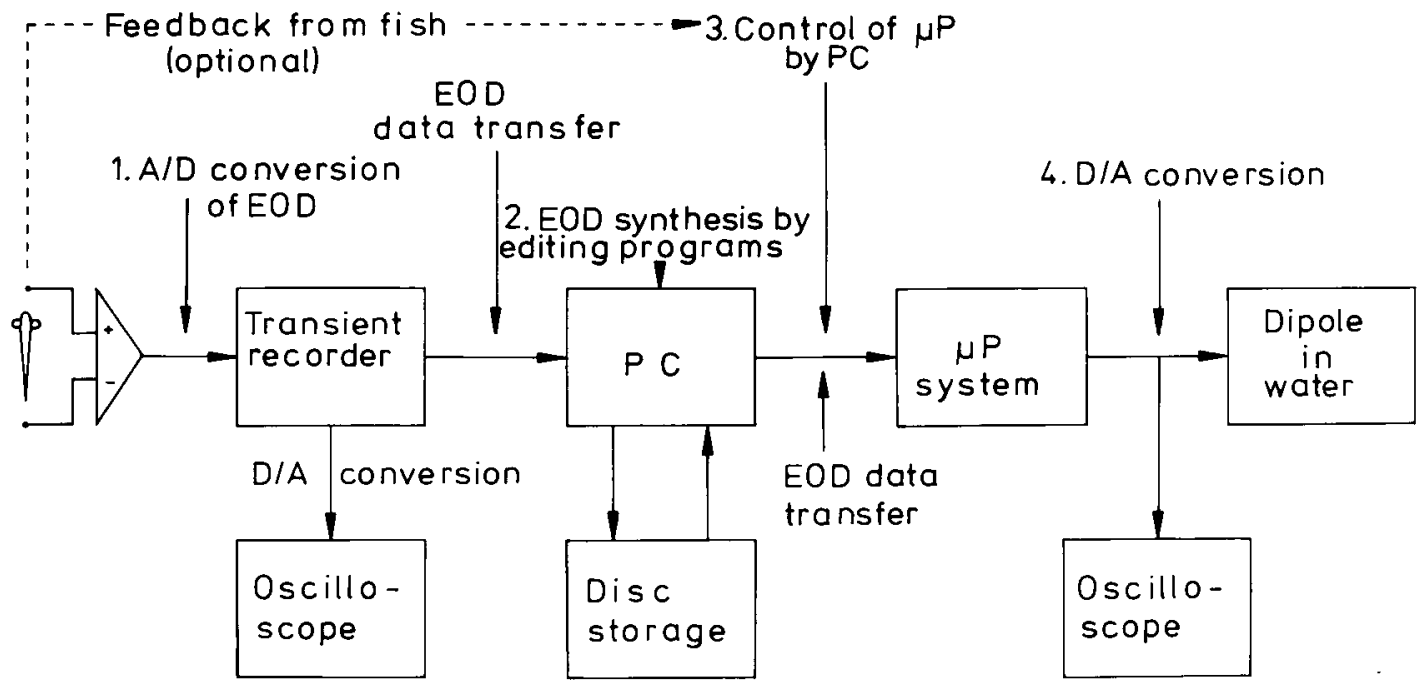

Fig. 4. Summary of the steps in EOD synthesis, as explained in the text. 


\section{Software}

The EOD synthesizer consists of an integrated program for the PC written in BASIC. It handles data input from the transient recorder, waveform synthesis, data storage and transfer to the $\mu \mathrm{P}$. Several application programs in BASIC prepare the $\mu \mathrm{P}$ for experiments during set-up, control the $\mu \mathrm{P}$ and other devices during experiments, analyze the data, etc.

\section{Basic performance objectives}

The basic objective in the design of our EOD synthesizer was the high-fidelity reproduction of EODs (or arithmetically modified EODs, or purely artificial waveforms). There is a trade-off between temporal and amplitude resolution in the choice of $\mathrm{A} / \mathrm{D}$ - and $\mathrm{D} / \mathrm{A}$-converters: we considered high temporal resolution (up to $500 \mathrm{kHz}$ ) of the utmost importance for the retainment of waveform, and an 8-bit amplitude resolution (256 linear levels) to be sufficient. The output is cyclically continuous in the case of wave EODs, and internally or externally triggered in the case of pulse EODs.

Wave EODs. Amplitude and the rate of $\mathrm{D} / \mathrm{A}$ conversion, hence frequency of the synthesized signal, are continuously variable on-line to the animal under test, enabling closed-loop experiments.

Pulse EODs. In addition to the features of wave EODs, the trigger mode includes (1) external triggering with fixed (but programmable) latency to the triggering pulses; (2) internal triggering from a list of time intervals, stored in the $\mu \mathrm{P}$ 's memory; (3) external triggering with internally specified delay times according to a list of time intervals, stored in the $\mu$ P's memory. All intervals are generated at $0.1-\mathrm{ms}$ resolution.

As the synthesis of pulse EODs is straightforward (see Introduction, and Figs. 1c and 2, bottom), the synthesis of wave EODs will receive special attention in the following.

\section{Steps in synthesis}

Four basic steps in the process of modification of sampled EODs for synthesis will be discussed using Eigenmannia's wave EOD (Figs. 5 and 6) as an example (a summary of this process is shown in Fig. 4).

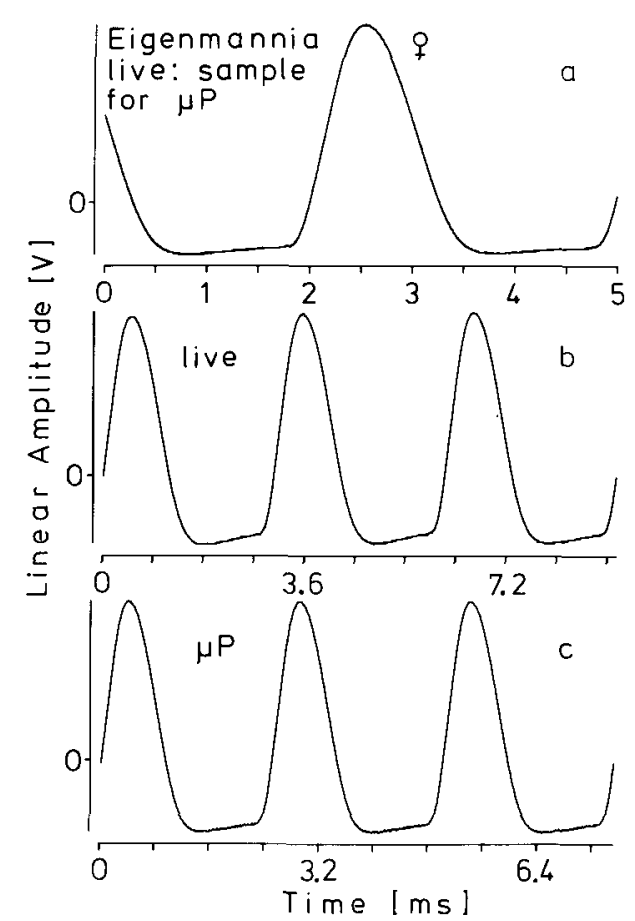

Fig. 5. Synthesis of the EOD of a female Eigenmannia lineata. a: the digitized sample of the EOD of the live fish which served for synthesis ( $400 \mathrm{kHz}$ digitization). b: the EOD of the live fish $(328 \mathrm{~Hz})$, digitized at $100 \mathrm{kHz}$ with 8 -bit resolution (water conductivity: $100 \mu \mathrm{S} / \mathrm{cm}$; temperature: $27.0^{\circ} \mathrm{C}$ ). c: the synthetic EOD $(400 \mathrm{~Hz})$ recorded from the $\mu \mathrm{P}$ output, digitized at $100 \mathrm{kHz}$ with 12-bit resolution.

$E O D$ input. The Vuko transient recorder registers input voltages in $2^{8}$ or 256 discrete linear levels. These levels are represented as integer numbers 0 to +255 for data transfer and arithmetic manipulations by computer programs. The sampling rate of the transient recorder is up to $2 \mathrm{MHz}$ or one digital representation of voltage every $0.5 \mu \mathrm{s}$. With the transient recorder's 2000 word memory, recording time is $1 \mathrm{~ms}$. This is less than one EOD cycle in Eigenmannia species which usually discharge at $250-500 \mathrm{~Hz}$ (or 2-4 ms per cycle). A sampling rate of $400 \mathrm{kHz}$ (2.5 $\mu$ s per step) gives a 5-ms recording time; this was sufficient for capturing at least one EOD cycle in all individuals (Figs. 5a, 6a). In an Apteronotus albifrons discharging at $870 \mathrm{~Hz}$, a sampling rate of $1 \mathrm{MHz}(1 \mu \mathrm{s}$ per step, that is $2 \mathrm{~ms}$ recording time) was used (not shown). The digitized samples were transferred as a block from the transient recorder memory to the computer and stored as disc files. 


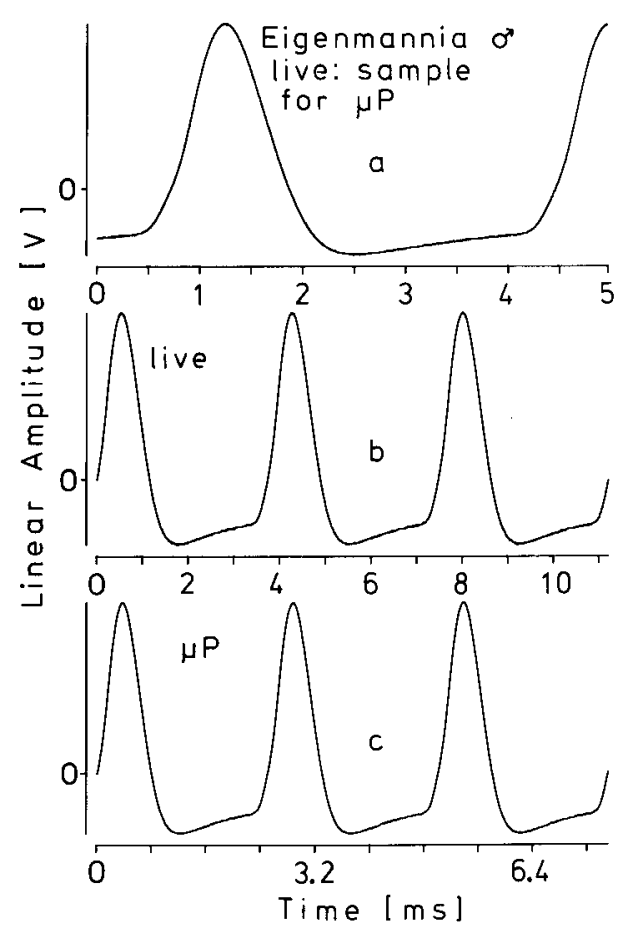

Fig. 6. Synthesis of the EOD of a male Eigenmannia lineata (as Fig. 5, but EOD frequency was $268 \mathrm{~Hz}$ ).

Arithmetic preparation of EOD sample for output. The editing program described below was designed to analyze Eigenmannia species (Figs. 5 and 6) and Apteronotus albifrons (see ref. 14) EODs; more complicated waveforms (for example, those with more than one maximum or minimum per cycle; see ref. 17) may require other algorithms.

As the desired maximum frequency of the synthesized EOD was greater than $244 \mathrm{~Hz}$, more than one EOD cycle (4 in these examples) had to be loaded into the working RAM. First, the editing program determines the length (that is, the start and the end locations) of one EOD cycle within the original A/D-data array. That portion of the array is digitally smoothed, arithmetically expanded to the full 8-bit range (that is, to a maximum value of 255 and a minimum value of 0 ), and redimensioned to an array of 512 elements (in the case of a desired maximum frequency of up to $976 \mathrm{~Hz}$ ). This array, representing one EOD cycle, is concatenated with itself 3 times in turn, so that a 2048-element array results, comprising 4 complete and identical EOD cycles. The whole array is coded as ASCII-characters (representing integer numbers from 0 to +255 ), stored on disc, and transferred to the $\mu \mathrm{P}$ memory.

A continuous signal is generated by the $\mu \mathrm{P}$ as explained above ( 3 cycles shown in Figs. $5 \mathrm{~b}$ and 6b). The number of cycles stored in the working RAM is relevant for the desired frequency range only. With 4 cycles stored, our system synthesizes EODs of a frequency of up to $976 \mathrm{~Hz}$ (frequency resolution is $0.24 \mathrm{~Hz}$ in that case). Instead of an EOD, any other periodic function may, of course, be synthesized.

Control of the $\mu P$ by the $P C$. After set-up of the $\mu \mathbf{P}$ for an experiment, several instructions have to be sent to the $\mu \mathrm{P}$ from the $\mathrm{PC}$ in order to specify a job (Fig. 3). These concern: (1) which waveform is to be used for the output ( 1 out of up to 7 stored in 7 separate memory areas); (2) amplitude (256 linear levels from 0 to full output at each attenuator setting); (3) envelope rise time $(0-2000 \mathrm{~ms})$ and (4) frequency. These parameters are sent to the $\mu \mathrm{P}$ as strings of ASCII characters via standard output commands in BASIC. They can be sent any time; the $\mu \mathbf{P}$ is under complete control of the PC (except during amplitude change when the $\mu \mathrm{P}$ will not accept commands or data). The PC can send new instructions on an internal or external interrupt (for example, from the experiment).

EOD output. The D/A converter (Precision Monolithic Instruments, PMI-DAC08) incorporated in our system (Fig. 3) is a fast (85 ns settling time) 8-bit device. Due to the frequency synthesizer, the maximum conversion rate is $500 \mathrm{kHz}$. The output of the $\mathrm{D} / \mathrm{A}$ converter is fed into a programmable (8-bit) output-amplifier with symmetrical outputs working in counter-phase (frequency response: $2 \mathrm{~Hz}-100 \mathrm{kHz}$; $\pm 24 \mathrm{~V}$ peak-to-peak full output at $0 \mathrm{~dB}$ attenuation). The two outputs each contain one manually selectable, mechanically coupled attenuator $(0-110 \mathrm{~dB}$ in 10 $\mathrm{dB}$ steps; output impedance: $600 \Omega$ ). At each attenuator setting the amplification can be programmed from 0 to full output in 256 linear levels.

Because of the symmetrical amplifier outputs the use of a stimulus isolation unit was unnecessary in our experiments. The signal can be applied directly to a dipole for the stimulation of 
fish. Best results (that is, minimum waveform distortion) are achieved by the use of carbon electrodes with large surface areas. For other applications, other transducers such as loudspeakers or vibrators may be used (with appropriate modification of the output amplifier).

The only difference between the synthetic EOD imitations produced by our $\mu \mathrm{P}$ (Figs. 1c, 2 (bottom), 5c, and 6c) and fish EODs (Figs. 1a, 2 (top, solid trace), 5b, and 6b) is that the synthetic EODs show stepwise voltage changes due to their digital storage and synthesis, while fish EODs, of course, do not. The voltage steps in the recordings of fish EODs are artifacts (digital noise) due to the A/D conversion. The amplitude of the digital noise of the synthesized signals is $<5 \%$ of the peak-to-peak signal amplitude $(0.4 \%$ of sine waves below $500 \mathrm{~Hz}$ ). The fish are certainly insensitive to that low-level, high-frequency digital noise, since the $\mathrm{D} / \mathrm{A}$ conversion rate of our $\mu \mathrm{P}$, depending on the desired maximum frequency of EOD synthesis and the actual output frequency, ranges from $250 \mathrm{kHz}$ to $500 \mathrm{kHz}$ in wave EODs $(500 \mathrm{kHz}$ in pulse EODs). Electroreceptors are tuned to the low frequency fundamental of wave EODs, that is, $250-650 \mathrm{~Hz}$ in Eigenmannia, and about $800-1100 \mathrm{~Hz}$ in Apteronotus ${ }^{8,20-22}$. In Eigenmannia the high frequency cut-off slope of single electroreceptor tuning curves is 55 $\mathrm{dB} /$ decade (or $17 \mathrm{~dB} /$ octave $^{22}$ ). In gymnotoid pulse fish electroreceptors are approximately tuned to the peak power frequency of the pulse $\mathrm{e}^{1,10}$ which is around $10 \mathrm{kHz}$ in extremely short pulse EODs, such as that of the mormyrid $P$. isidori $^{11}$.

\section{APPLICATIONS}

Eigenmannia's jamming avoidance response $\left(\mathrm{JAR}^{23}\right)$ is a frequency shift away from a similar frequency of a close neighbour. The assumed function of the response is an improvement of the fish's signal-to-noise ratio for better electrolocation in the presence of a neighbour's jamming signal $^{5,23}$. Since at least two fish are involved under natural conditions this behaviour has been qualified as communication behaviour ${ }^{3,6,20}$.

Gottschalk $^{4}$ considered a temporal feature of the EOD wave-form based on the intervals between zero-crossings of prime importance for signal detection and jamming avoidance behaviour (and, by implication, also for species and mate recognition). In order to test how Eigenmannia detects and analyzes stimulus waves, an on-line system affording free choice of stimulus waveforms, amplitudes, and frequencies was needed, such as the one presented here. The results of closed-loop JAR experiments, using various artificial and synthetic 'natural' waveforms, show that $E$. lineata performs the equivalent of a limited Fourier amplitude spectrum analysis of the stimulus signal, and is insensitive to waveform in the context of the JAR. Eigenmannia assesses the intensity of that harmonic signal component which is close to its own EOD fundamental frequency, showing that the mechanism of signal analysis involved is a frequency domain mechanism ${ }^{15}$. This has been tested, for example, by the use of digitally synthesized male and female EODs (see Figs. 5 and 6, and Fig. 11 in ref. 15 which also gives Fourier amplitude spectra), and by purely artificial signals of identical amplitude spectra, synthesized by computer (Fig. 9 in ref. 15). The waveforms of the latter signals were dissimilar due to different phase relationships among their harmonics.

However, Eigenmannia does discriminate between the male and female EOD waveforms as confirmed in a food-rewarded, conditioned discrimination experiment. With stimuli presented in randomized sequence, each of $4 E$. lineata clearly discriminated the rewarded synthetic female EOD from the unrewarded synthetic male EOD $^{19}$. The animals also discriminated sawtooth waves from sine waves, and synthetic male EODs from sine waves. The fish quickly changed their preference when the roles of the rewarded and unrewarded waveforms were reversed. The fish still discriminated successfully between sawtooth and sine wave signals when the intensities of their fundamental frequencies were equal instead of their peak-to-peak amplitudes, excluding a purely intensity-dependent discrimination. The pairs of signals which were discriminated differed markedly both in waveform and harmonic content.

The aim of experiments in progress is to find out more about the mechanisms of this discrimi- 
nation using our microprocessor-based system which affords free choice among natural and artificial waveforms over a wide range of frequencies and amplitudes.

\section{ACKNOWLEDGEMENTS}

This work was supported by the Deutsche Forschungsgemeinschaft (SFB4/H1). We wish to thank Prof. H. Markl (University of Konstanz) for suggesting that this report be written and Prof. P.M. Narins (University of California at Los Angeles, UCLA) and B.O. Bratton, as well as two anonymous referees for critically reading the manuscript and giving many helpful suggestions.

\section{REFERENCES}

1 Bastian, J., Frequency response characteristics of electroreceptors in weakly electric fish (Gymnotoidei) with a pulse discharge, J. Comp. Physiol., 112 (1976) 165-180.

2 Bullock, T.H., Electroreception, Ann. Rev. Neurosci., 5 (1982) 121-170.

3 Bullock, T.H., Hamstra, R.H. and Scheich, H., The jamming avoidance response of high frequency electric fish. I. General features. II. Quantitative aspects, $J$. Comp. Physiol., 77 (1972) 1-48.

4 Gottschalk, B., Electrocommunication in gymnotid wave fish: significance of a temporal feature in the electric organ discharge. In T. Szabo and G. Czeh (Eds.), Sensory Physiology of Aquatic Lower Vertebrates, Adv. Physiol. Sci., Vol. 31, Pergamon Press, Budapest, 1981, pp. 255-277.

5 Heiligenberg, W., Principles of Electrolocation and Jamming Avoidance in Electric Fish. Studies of Brain Function, Vol. 1. Springer, Berlin, 1977, 85 pp.

6 Heiligenberg, W., The jamming avoidance response in the weakly electric fish Eigenmannia. A behavior controlled by distributed evaluation of electroreceptive afferences, Naturwissenschaften, 67 (1980) 499-507.

7 Heiligenberg, W. and Altes, R.A., Phase sensitivity in electroreception, Science, 199 (1978) 1001-1004.

8 Hopkins, C.D., Stimulus filtering and electroreception: tuberous electroreceptors in three species of gymnotoid fish, J. Comp. Physiol., 111 (1976) 171-207.

9 Hopkins, C.D., and Bass, A.H., Temporal coding of species recognition signals in an electric fish, Science, 212 (1981) 85-87.

10 Hopkins, C.D., and Heiligenberg, W.F., Evolutionary designs for electric signals and electroreceptors in gymnotoid fishes of Surinam, Behav. Ecol. Sociobiol, 3 (1978) 113-134.

$11 \mathrm{Kramer}, \mathrm{B} .$, Spontaneous discharge rhythms and social signalling in the weakly electric fish Pollimyrus isidori
(Cuvier et Valenciennes) (Mormyridae, Teleostei), Behav. Ecol. Sociobiol., 4 (1978) 61-74.

12 Kramer, B., Electric and motor responses of the weakly electric fish, Gnathonemus petersii (Mormyridae)), to playbacks of social signals, Behav. Ecol. Sociobiol., 6 (1979) 67-79.

13 Kramer, B., Electrocommunication in fish: stimulus waveform-dependent responses in Eigenmannia (Gymnotiformes, Teleostei) - a model for intraspecific communication? Verh. Dtsch. Zool. Ges. 1983, 170 (in German).

14 Kramer, B., Tranquillizer reduces electric organ discharge frequency in a teleost fish, Naturwissenschaften, 71 (1984) 99.

$15 \mathrm{Kramer}$, B., Jamming avoidance in the electric fish Eigenmannia: harmonic analysis of sexually dimorphic waves, J. Exp. Biol., 119 (1985) 41-69.

16 Kramer, B., Kommunikation mit elektrischen Signalen bei Fischen. In D. Franck (Ed.), Verhaltensbiologie, 2nd edn., Deutscher Taschenbuch, Thieme, Stuttgart, 1985, pp. 273-277.

17 Kramer, B., Kirschbaum, F. and Markl, H., Species specificity of electric organ discharges in a sympatric group of gymnotoid fish from Manaus (Amazonas). In T. Szabo and G. Czeh (Eds.), Sensory Physiology of Aquatic Lower Vertebrates, Adv. Physiol. Sci., Vol. 31, Pergamon Press, Budapest, 1981, pp. 195-219.

$18 \mathrm{Kramer}$, B. and Westby, G.W.M., No sex difference in the waveform of the pulse type electric fish, Gnathonemus petersii (Mormyridae), Experientia, 41 (1985) 1530-1531.

19 Kramer, B. and Zupanc, G.K.H., Conditioned discrimination of electric waves differing only in form and harmonic content in the electric fish, Eigenmannia, Naturwissenschaften, in press.

20 Scheich, H., Neural basis of communication in the high frequency electric fish, Eigenmannia virescens (jamming avoidance response). I. Open loop experiments and the time domain concept of signal analysis. II. Jammed electroreceptor neurons in the lateral line nerve. III. Central integration in the sensory pathway and control of the pacemaker, J. Comp. Physiol., 113 (1977) 181-255.

21 Scheich, H., Bullock, T.H. and Hamstra Jr., R.H. Coding properties of two classes of afferent nerve fibers: high frequency electroreceptors in the electric fish, Eigenmannia, J. Neurophysiol., 36 (1973) 39-60.

22 Viancour, T.A., Electroreceptors of a weakly electric fish. I. Characterization of tuberous electroreceptor tuning. II. Individually tuned receptor oscillations, J. Comp. Physiol., 133 (1979) 317-338.

23 Watanabe, A. and Takeda, K., The change of discharge frequency by a.c. stimulus in a weak electric fish, J. Exp. Biol., 40 (1963) 57-66.

24 Westby, G.W.M., Assessment of the signal value of certain discharge patterns in the electric fish, Gymnotus carapo, by means of playback, J. Comp. Physiol., 92 (1974) 327-341. 